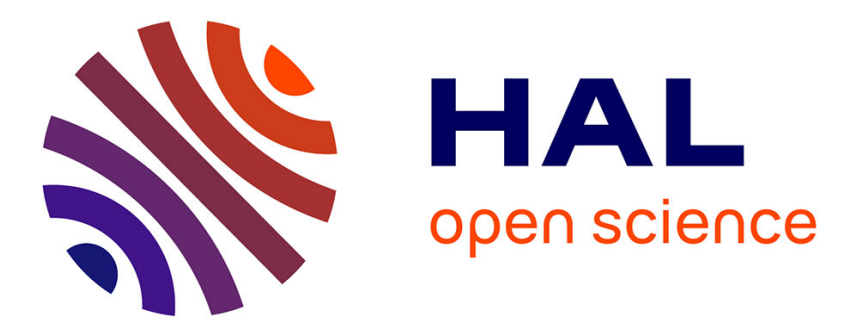

\title{
MALICE : un logiciel couplant la méthode intégrale et la méthode des éléments finis. Application à la fusion des oxydes en creuset froid
}

\author{
S. Servant, D. Saumabere, A. Gagnoud, Y. Du Terrail Couvat
}

\section{To cite this version:}

S. Servant, D. Saumabere, A. Gagnoud, Y. Du Terrail Couvat. MALICE : un logiciel couplant la méthode intégrale et la méthode des éléments finis. Application à la fusion des oxydes en creuset froid. Journal de Physique III, 1992, 2 (11), pp.1991-2004. 10.1051/jp3:1992225 • jpa-00248860

\section{HAL Id: jpa-00248860 https://hal.science/jpa-00248860}

Submitted on 1 Jan 1992

HAL is a multi-disciplinary open access archive for the deposit and dissemination of scientific research documents, whether they are published or not. The documents may come from teaching and research institutions in France or abroad, or from public or private research centers.
L'archive ouverte pluridisciplinaire HAL, est destinée au dépôt et à la diffusion de documents scientifiques de niveau recherche, publiés ou non, émanant des établissements d'enseignement et de recherche français ou étrangers, des laboratoires publics ou privés. 


\title{
MALICE : un logiciel couplant la méthode intégrale et la méthode des éléments finis. Application à la fusion des oxydes en creuset froid
}

\author{
S. Servant, D. Saumabere, A. Gagnoud et Y. du Terrail Couvat \\ Laboratoire MADYLAM, ENSHMG, B.P. 95, 38042 Saint-Martin d'Hères, France \\ (Reçu le 23 mars 1992, révisé le 3 juillet 1992, accepté le 10 août 1992)
}

\begin{abstract}
Résumé. - Le logiciel MALICE modélise les phénomènes physiques couplés dans les installations d'élaboration par induction. Il est le résultat du couplage de deux logiciels : OPHELIE et FLUXEXPERT. Le phénomène électromagnétique est résolu par la Méthode Intégrale (MI), bien adaptée au problème électromagnétique $3 \mathrm{D}$ notamment pour les domaines infinis et les régions où l'épaisseur de peau électromagnétique est faible. Ceci est particulièrement intéressant pour la modélisation du creuset froid. Le logiciel FLUX-EXPERT est basé sur la Méthode des Eléments Finis (MEF) et contient un générateur d'équations incluant la description des phénomènes thermique et hydrodynamique. Les résultats obtenus dans le cadre de la fusion des oxydes en creuset froid mettent en évidence l'intérêt du couplage. Les propriétés physiques des oxydes varient fortement avec la température et l'interaction entre les phénomènes est importante. Les résultats obtenus sans couplage sont erronés et peuvent conduire à un dimensionnement aberrant de l'installation de fusion.
\end{abstract}

\begin{abstract}
The multimethod Malice package allows to modelize coupled phenomena in the induction elaboration. It is the result of the coupling of 3D electromagnetic package OPHELIE and a generator of partial derivative equations FLUX EXPERT. The electromagnetic model is solved by integral method. It is well adapted to 3D problem, infinite domain of study and fine skin depth which is particularly interesting for the modelling of cold crucible installation. FLUX EXPERT uses finite element method to solve phenomenon equations: thermic and hydrodynamic phenomena are described in its equation base. In the particular application of fusion of oxyde in cold crucible, the results obtained enhance the interest of the package. The physical properties of these materials strongly depend on temperature, thus physical phenomena are coupled. Results calculated without coupling may lead to false exploitation of the process.
\end{abstract}

\section{Introduction.}

Le logiciel MALICE a été conçu pour modéliser les phénomènes physiques intervenant dans les procédés d'élaboration en creuset froid inductif. Un inducteur, parcouru par un courant alternatif sinusoïdal, crée un champ magnétique dans lequel est placé un creuset de cuivre sectorisé refroidi par une circulation interne d'eau. Des courants induits se développent en 
superficie des secteurs. L'originalité du procédé repose sur la sectorisation qui empêche le creuset de faire écran au champ magnétique. Les courants induits dans le matériau à élaborer sont à l'origine de deux effets :

- un effet thermique : chauffage et fusion du matériau par effet Joule,

- un effet mécanique : création, sous l'action des forces de Laplace, d'un brassage du bain liquide.

L'analyse des phénomènes électromagnétique, thermique et hydrodynamique intervenant dans ce procédé met en évidence leur interdépendance. Aussi seule une résolution couplée des équations constitutives permet de modéliser la réalité. De plus les domaines d'étude sont différents d'un phénomène physique à l'autre :

- il est tridimensionnel et infini pour l'électromagnétisme,

- il est limité à la charge axisymétrique pour la thermique et l'hydrodynamique.

Une modélisation complète du procédé par la méthode des élements finis conduit à des systèmes non linéaires de taille très importante car la géométrie est tridimensionnelle et le nombre d'inconnues par nœud élevé [1]. En conséquence la démarche adoptée a été de choisir la méthode numérique la mieux adaptée à chaque phénomène physique.

Ainsi pour la résolution du phénomène électromagnétique tridimensionnel nous avons choisi la méthode intégrale [2]. Le code utilisé est OPHELIE développé à MADYLAM.

Pour les phénomènes thermique et hydrodynamique nous avons choisi la méthode des éléments finis. Le logiciel utilisé est FLUX EXPERT. I1 possède un générateur de code éléments finis permettant la simulation des formulations obtenues par méthode projective des équations aux dérivées partielles [3].

MALICE est le logiciel couplant la méthode intégrale (OPHELIE) et la méthode des éléments finis (FLUX EXPERT). Ce code offre la possibilité de sélectionner les phénomènes physiques à résoudre en couplage avec l'électromagnétisme (thermique, hydrodynamique.. ). La difficulté de son élaboration consiste à réaliser la liaison des termes de couplage d'un maillage à l'autre puis de construire un jeu d'algorithmes de résolution itérative permettant de traiter des problèmes en régime transitoire ou permanent du cycle d'élaboration.

L'ensemble des applications de l'élaboration en creuset froid inductif peut être modélisé par MALICE : élaboration statique de matériaux en creuset de type poche, refusion de barreaux ou copeaux pour tirer en continu des lingots en creuset droit.

En fonction du matériau étudié, les phénomènes physiques prépondérants et le type de résolution adaptés peuvent être sélectionnés :

- pour les matériaux métalliques, l'écoulement dans le bain est turbulent. Il importe alors d'associer hydrodynamique et thermique. Un algorithme de résolution séquentiel des phénomènes électromagnétique et thermodynamique est suffisant, la conductivité électrique variant peu avec la température;

- pour les oxydes, la résolution couplée de l'électromagnétisme et de la thermique est indispensable et un algorithme non linéaire est choisi car les propriétés physiques des oxydes varient fortement avec la température.

C'est ce dernier type d'exemple que nous avons choisi de présenter afin d'illustrer les possibilités de ce logiciel et notamment l'intérêt du couplage fort électromagnétique-thermique. Pour la fusion d'oxydes en creuset froid (Fig. 1), la forte variation des propriétés en fonction de la température $[4,5]$ rend le système non linéaire. Par contre les forces de Laplace, origine du brassage pour les métaux, sont faibles. Le mouvement du bain est uniquement dû à la thermo-convection. Etant donné l'importance de la viscosité du matériau nous négligerons dans un premier temps l'hydrodynamique. 


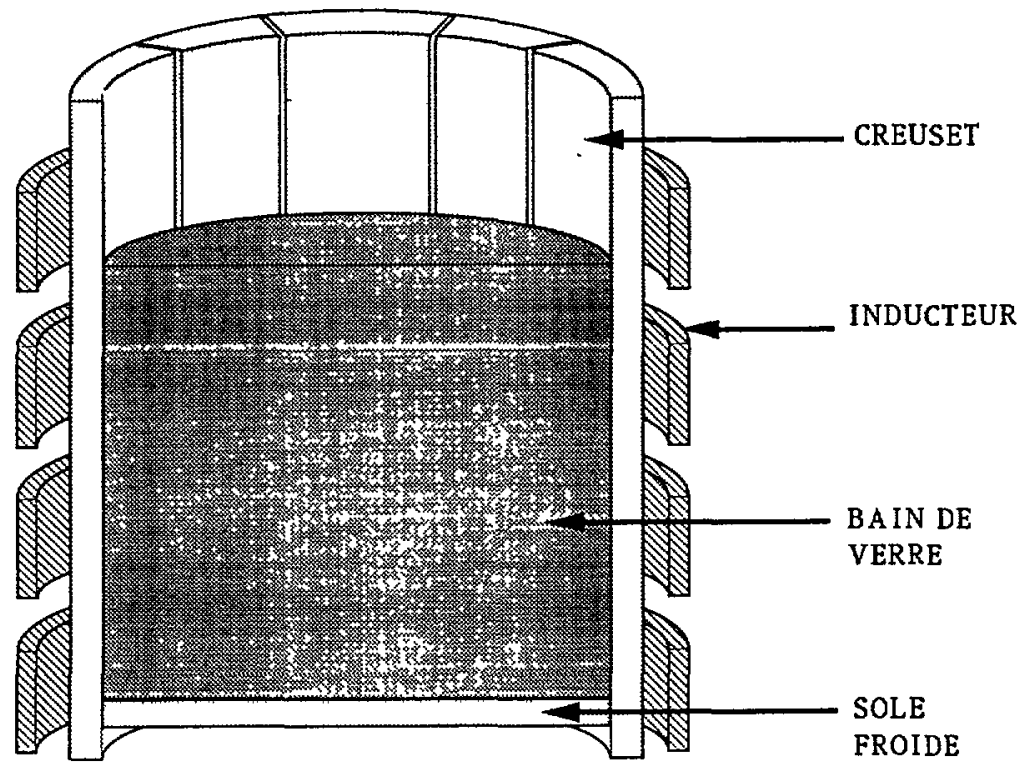

Fig. 1. - Schéma de l'installation de fusion de verres en creuset froid.

[Schematic representation of the glass fusion in cold crucible.]

\section{Modélisation.}

1.1 MOdèle ÉleCtromagnétiQue. - Le phénomène caractéristique de l'induction dans un matériau conducteur de l'électricité est la notion de diffusion du champ magnétique dans le matériau. L'équation correspondante s'écrit :

$$
\Delta \mathbf{B}=\mu \sigma \frac{\partial \mathbf{B}}{\partial t}
$$

où $\mathbf{B}$ est l'induction magnétique, $\mu$ est la perméabilité magnétique, $\sigma$ est la conductivité électrique et $t$ est le temps.

L'induction magnétique variant sinusoïdalement avec le temps cette équation met en évidence la longueur caractéristique de diffusion du champ magnétique dans le matériau, appelée épaisseur de peau électromagnétique $\delta$,

$$
\delta=\sqrt{2 / \mu \sigma \omega}
$$

où $\omega$ est la pulsation du courant inducteur.

Lorsque l'épaisseur de peau électromagnétique est faible devant les dimensions du matériau, les grandeurs électromagnétiques varient selon une loi de décroissance exponentielle en fonction de la distance à la surface. Pour les fréquences habituelles de fonctionnement de ce type d'installation ( $1 \mathrm{kHz}-500 \mathrm{kHz}$ ) l'épaisseur de peau est faible dans le creuset, l'inducteur et la sole. Elle est grande dans les oxydes.

Une résolution par la méthode des éléments finis nécessite des maillages complexes et lourds. Le nombre d'inconnues par nœud est alors de quatre (les trois composantes du potentiel vecteur et le potentiel électrique). La méthode choisie est la méthode intégrale, mieux adaptée 
à ce problème car elle utilise un maillage limité aux parties électriquement actives et réduit de plus le nombre d'inconnues à deux (une composante de la densité de courant et le potentiel électrique). Cette méthode permet d'optimiser la taille du maillage.

Les équations résolues par la méthode intégrale sont :

$$
\begin{gathered}
\operatorname{div} \mathbf{J}=0 \\
\oint \operatorname{grad} V \cdot \mathrm{d} \mathbf{l}=0 \\
\mathbf{J}=-\sigma\left(\operatorname{grad} V+\frac{\partial \mathbf{A}}{\partial t}\right)
\end{gathered}
$$

où $\mathbf{A}$ est le potentiel vecteur, $V$ est le potentiel scalaire, $\mathbf{J}$ est la densité de courant.

Le potentiel vecteur s'exprime par la loi de Biot et Savart qui prend naturellement en compte les conditions aux limites à l'infini.

Le creuset froid est de géométrie tridimensionnelle, périodique en $\theta$ (direction azimutale) et les autres éléments (charge, sole et inducteur) sont axisymétriques. Le maillage (charge, sole, creuset, inducteur) est constitué d'un ensemble de courants élémentaires de direction imposée (Fig. 2). De plus, la prise en compte de la loi de décroissance exponentielle dans la formulation
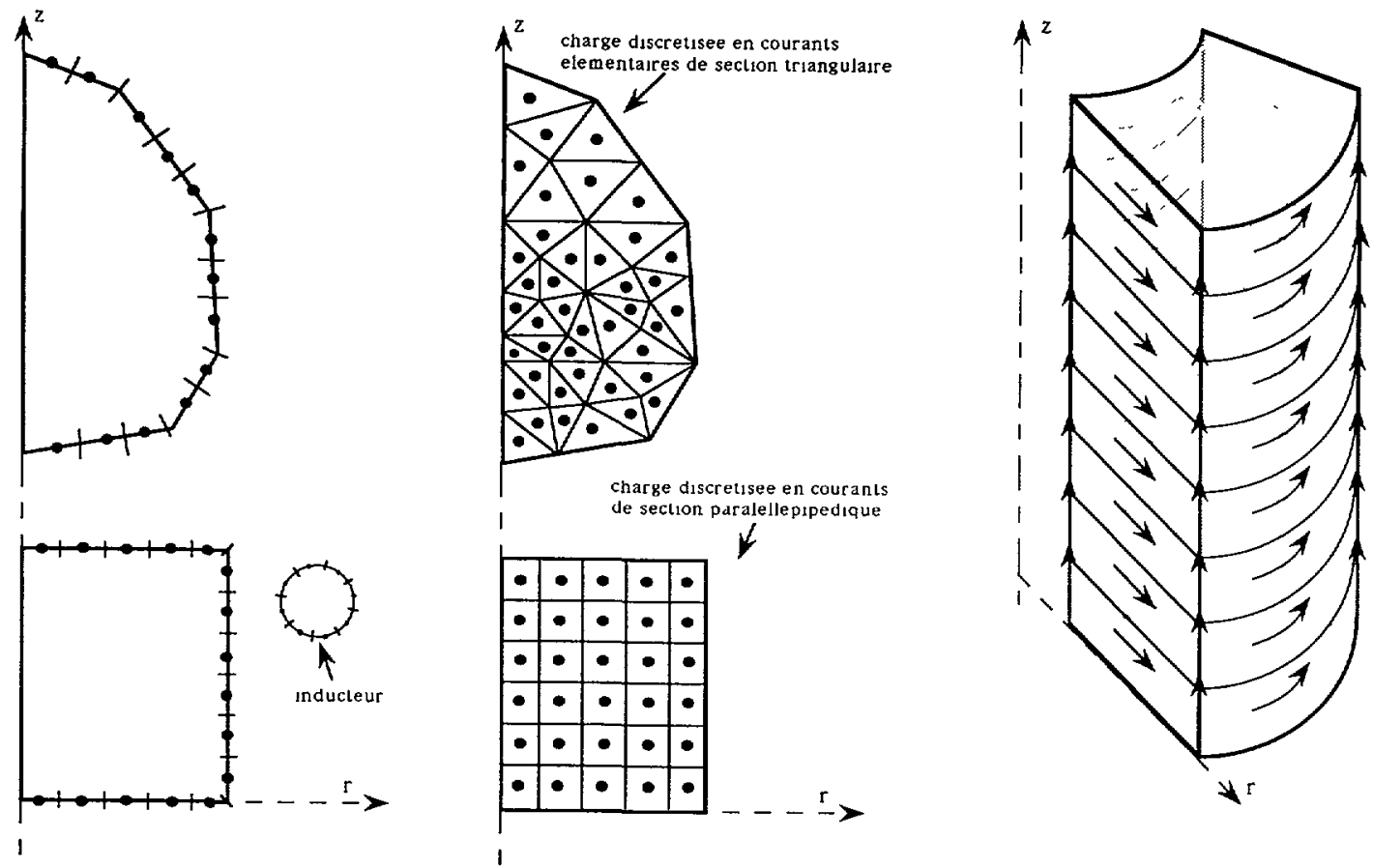

- noeuds de calcul des courants industs orientes suivant la direction azimutale

Trace des courants elementates a la surface d un secteur de creuset

Fig. 2. - Maillage électromagnétique : a) charge axisymétrique, hypothèse de fine épaisseur de peau électromagnétique, maillage surfacique ; b) charge axisymétrique, hypothèse de grande épaisseur de peau électromagnétique, maillage volumique ; $c$ ) secteur de creuset froid, hypothèse de fine épaisseur de peau électromagnétique, maillage surfacique.

[Electromagnetic mesh : a) axisymmetric load, hypothesis of thin electromagnetic skin depth, superficial mesh ; b) axisymmetric load, hypothesis of large electromagnetic skin depth, volumic mesh ; c) sector of cold crucible, hypothesis of thin electromagnetic skin depth, superficial mesh.] 
permet de réduire le maillage à la surface des éléments conducteurs d'électricité : creuset, inducteur et sole. Le système obtenu est linéaire. Les inconnues sont la densité de courant et le potentiel électrique. La matrice est pleine et dissymétrique.

A partir des densités de courants induits calculées dans la charge, les densités de puissance Joule et les densités de force de Laplace sont calculées.

1.2 Modèle thermique. - Nous avons choisi d'exprimer la conservation de l'énergie en utilisant la formulation en température dont l'écriture générale en régime permanent est :

$$
\rho(T) C_{\mathrm{p}}(T) \mathbf{U} \cdot \operatorname{grad} T=\operatorname{div}(k(T) \operatorname{grad} T)+Q_{\mathrm{th}}+\Delta H
$$

où $\rho(T)$ est la densité, $C_{\mathrm{p}}(T)$ est la capacité calorifique, $T$ est la température, $\mathbf{U}=\mathbf{U}_{\mathrm{b}}+\mathbf{U}_{\mathrm{t}}$ est la somme de la vitesse du fluide et de la vitesse de tirage, $k(T)$ est la conductivité thermique, $Q_{\text {th }}$ est la densité de puissance dissipée par effet Joule, $Q_{\text {th }}=J J * / \sigma$, et $\Delta H$ est la chaleur latente de changement d'état.

La charge élaborée a une géométrie axisymétrique. La formulation éléments finis est bidimensionnelle axisymétrique [6]. Les conditions aux limites sont représentées sur la figure 3. A la surface de la charge on prend en compte le rayonnement à l'infini et la convection. La densité surfacique de puissance dissipée ainsi s'exprime :

$$
P=\varepsilon_{\mathrm{r}} \sigma_{\mathrm{sb}}\left(T_{\mathrm{s}}^{4}-T_{\mathrm{a}}^{4}\right)+h_{\mathrm{s}}\left(T_{\mathrm{s}}-T_{\mathrm{a}}\right)
$$

où

$\varepsilon_{\mathrm{r}} \quad$ est l'émissivité du matériau constituant la charge

$\sigma_{\text {sb }}$ est la constante de Stefan Boltzmann

$T_{\mathrm{s}} \quad$ est la température de surface du matériau

$T_{\mathrm{a}}$ est la température ambiante

$h_{\mathrm{s}}$ est le coefficient d'échange à la surface.

Le contact entre le creuset froid et la charge et celui entre la sole et la charge est un contact imparfait. La puissance ainsi dissipée est assimilée à un flux convectif :

$$
P=h\left(T_{\mathrm{c}}-T_{\mathrm{f}}\right)
$$

où $T_{c}$ est la température de la charge

et $T_{\mathrm{f}}$ est la température de la paroi froide de la sole ou du creuset.

Les propriétés du matériau dépendant de la température, le système obtenu est non linéaire. Il est résolu par la méthode de Newton-Raphson.

1.3 Couplage. - Le couplage concerne le domaine de la charge où toutes les inconnues du problème sont à déterminer. Les termes couplant les équations sont $\sigma(T)$ et $J J^{*} / \sigma$, conductivité électrique et densité de puissance Joule.

Le maillage de la charge utilisé dans la méthode intégrale est différent du maillage éléments finis de la charge. Des interpolations sont nécessaires pour calculer les termes de couplage. Les difficultés de ces interpolations sont de deux natures :

- Le choix de la grandeur à interpoler : certains termes de couplage sont des fonctions non linéaires de la variable d'état, par exemple $J J^{*} / \sigma=f(A)$. Ce sont les variables d'état température et potentiel vecteur qui sont interpolées d'un maillage à l'autre, les fonctions étant calculées ensuite localement. 


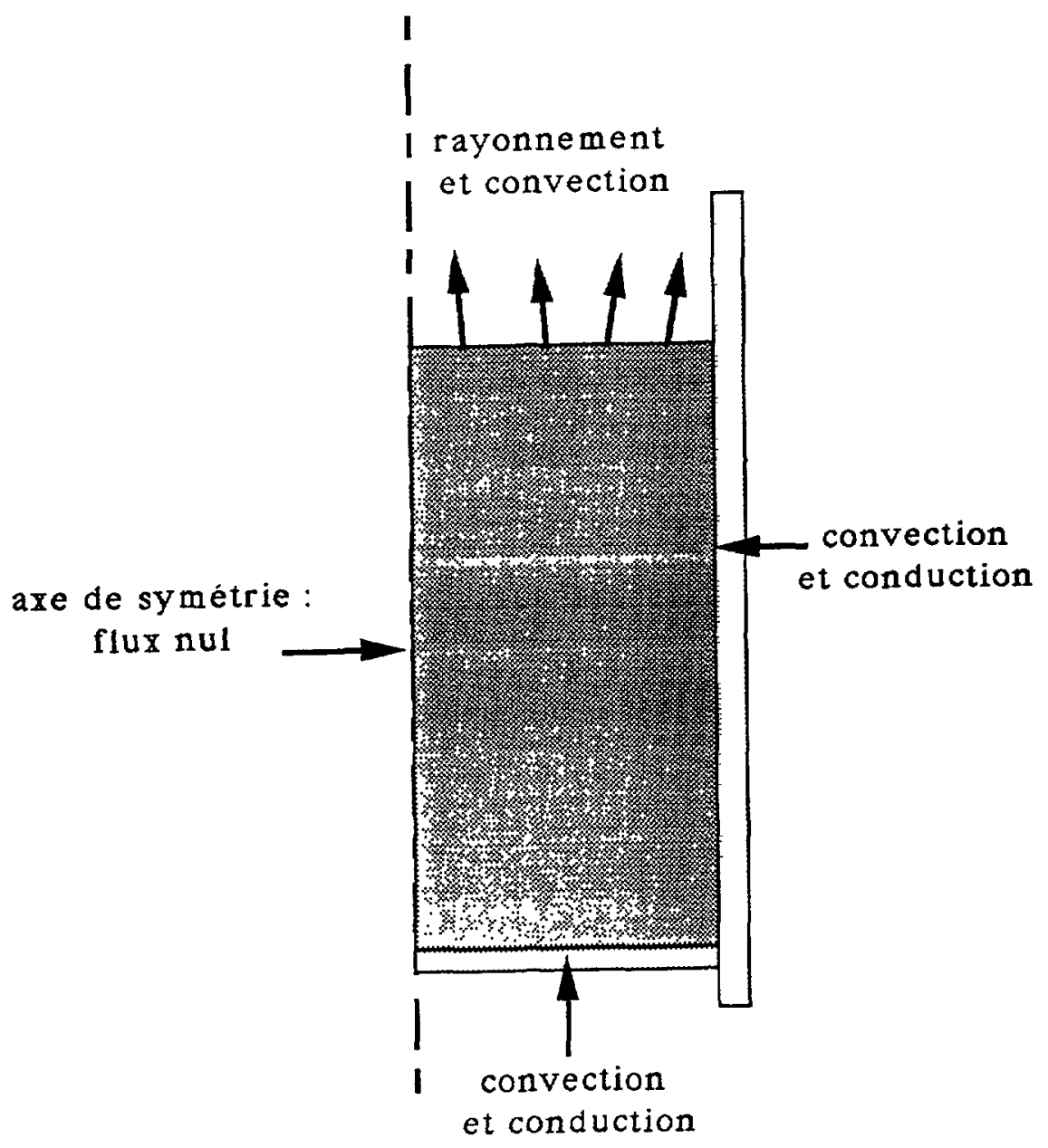

Fig. 3. - Conditions aux limites en thermique.

[Thermic boundary conditions.]

- La démarche de la localisation : la solution la plus rapide consiste à localiser les nœuds d'un maillage par rapport à l'autre, ceux-ci étant moins nombreux que les points d'intégration. Les grandeurs sont ensuite interpolées dans la maille aux points d'intégration. La solution la plus précise consiste à localiser directement les points d'intégration.

Les solutions retenues pour l'interpolation des termes de couplage sont :

interpolation $\mathrm{MI} \rightarrow \mathrm{MEF}$

1) localisation des points d'intégration du maillage MEF dans le maillage $\mathrm{MI}$,

2) interpolation du potentiel vecteur aux points d'intégration du maillage MEF et calcul des sources thermiques;

interpolation $\mathrm{MEF} \rightarrow \mathrm{MI}$

1) localisation des nœuds du maillage MI dans le maillage MEF,

2) calcul de la température puis de $\sigma(T)$ par interpolation aux nœuds du maillage MI. 
Dans le cadre de l'application aux verres (grande épaisseur de peau), l'interpolation est effectuée entre deux maillages volumiques. Dans ce cas on considère le maillage de la méthode intégrale comme un maillage éléments finis secondaire.

Lorsque la charge est conductrice d'électricité (fine épaisseur de peau), l'interpolation du potentiel vecteur est réalisée d'un maillage surfacique vers un maillage volumique. Dans ce cas pour tous les points d'intégration du maillage éléments finis on recherche le projeté orthogonal de ce point sur la surface ; le projeté orthogonal est localisé dans le maillage de la méthode intégrale et une loi de décroissance exponentielle suivant la normale à la surface est appliquée à la grandeur.

\section{Code.}

Un algorithme commandant les deux modules de résolution basés respectivement sur la méthode intégrale et sur la méthode des éléments finis a été créé (Fig. 4). Il comporte deux niveaux de résolution itérative imbriqués :

- la résolution du système non linéaire obtenu par la formulation éléments finis pour le phénomène thermique (Fig. 4) ;

— la boucle de résolution du système couplé méthode intégrale (électromagnétique) et méthode des éléments finis.

Deux préprocesseurs distincts permettent de décrire les domaines d'étude par la méthode intégrale et la méthode des éléments finis. Un module unique de résolution permet de calculer les grandeurs électromagnétique et thermique... Deux post-processeurs distincts ont été conservés afin d'optimiser l'exploitation des grandeurs calculées (Fig. 5).

\section{Résultats.}

L'illustration présentée est destinée à démontrer l'importance de tels couplages dans des résolutions multi-méthodes, multi-phénomènes. La configuration de fusion d'oxydes en creuset froid étudiée est représentée figure 6. Le modèle est appliqué au cas de la fusion statique $\left(u_{t}=0\right)$ en régime permanent. L'écoulement thermoconvectif est modélisé par une conductibilité thermique équivalente $\left(\mathbf{u}_{\mathrm{b}}=\mathbf{0}\right.$, kéquivalente $>$ kmoléculaire). Dans la modélisation, le creuset froid est de géométrie tridimensionnelle, périodique en $\theta$. La charge de verre, l'inducteur et la sole sont décrits par une géométrie axisymétrique. Sur la figure 5 est présenté le maillage électromagnétique. Le maillage du creuset, de l'inducteur et de la sole sont surfaciques. Le maillage du verre est volumique. Le maillage thermique est réduit à la région occupée par le verre (Fig. 7). Les modèles de propriétés sont tracés sur la figure 8 .

Nous présentons les résultats obtenus pour diverses valeurs de courant imposé dans l'inducteur. Afin de mettre en évidence la nécessité du couplage, chaque cas est modélisé par une résolution séquentielle unique électromagnétique-thermique (cas noté a) et par une résolution itérative (cas noté b).

Si les paramètres d'initialisation du problème (conductivité électrique, température, courant inducteur) sont proches des conditions de fonctionnement en régime permanent, les résultats sont du même ordre de grandeur dans les cas a et b (Fig. 9).

Si le courant de l'inducteur est sous-dimensionné, le bain de verre doit normalement se figer. $\mathrm{Au}$ contraire, la résolution sans couplage montre un échauffement du bain (Fig. 10a). La résolution couplée permettant le réajustement des sources de chaleur (les densités de puissance) à chaque itération, le figeage du verre est obtenu (Fig. 10b).

Le problème reste le même dans le cas où le courant de l'inducteur est trop important.

Le couplage permet de prendre en compte l'effet des conditions aux limites sur la répartition des sources de puissances Joule. En l'absence de couplage, la répartition des densités de 


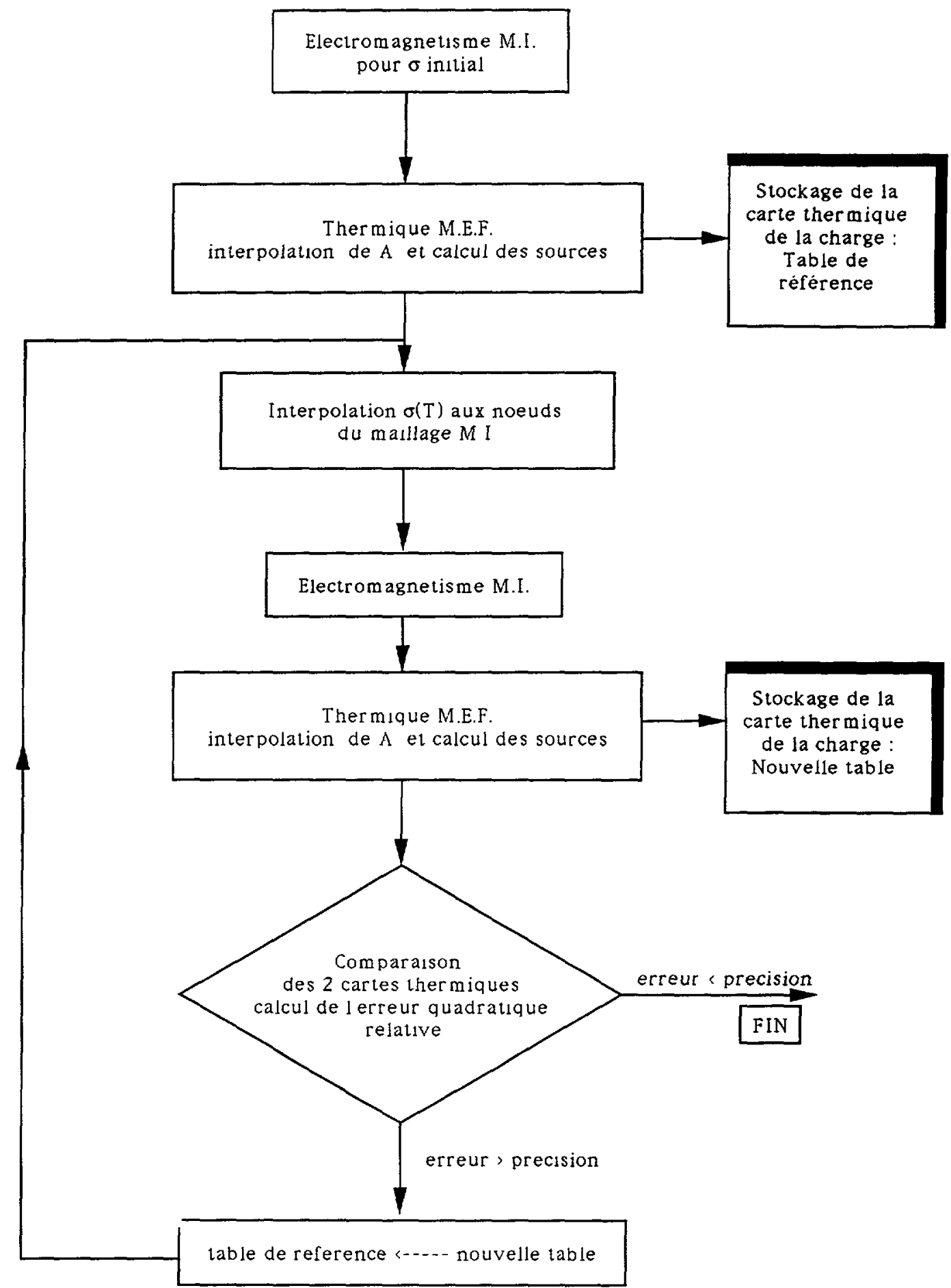

Fig. 4. - Algorithme de résolution.

[Resolution algorithm.] 


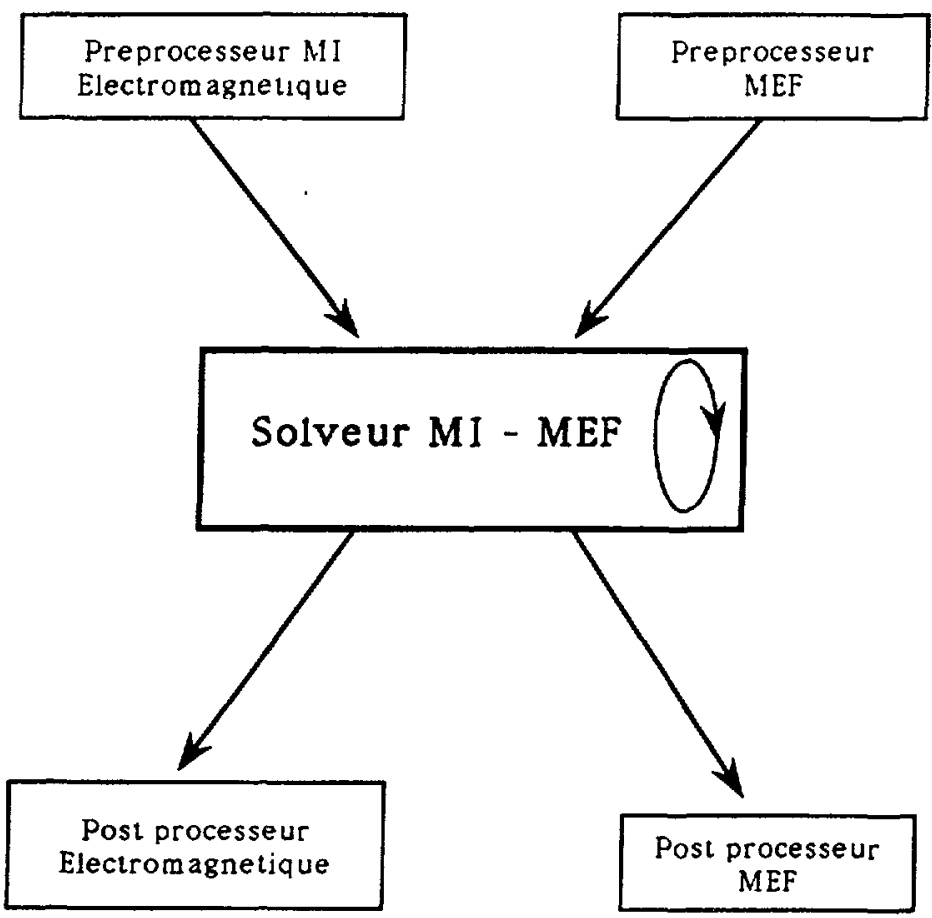

Fig. 5. - Principe d'utilisation du logiciel.

[Princip of the package utilisation.]

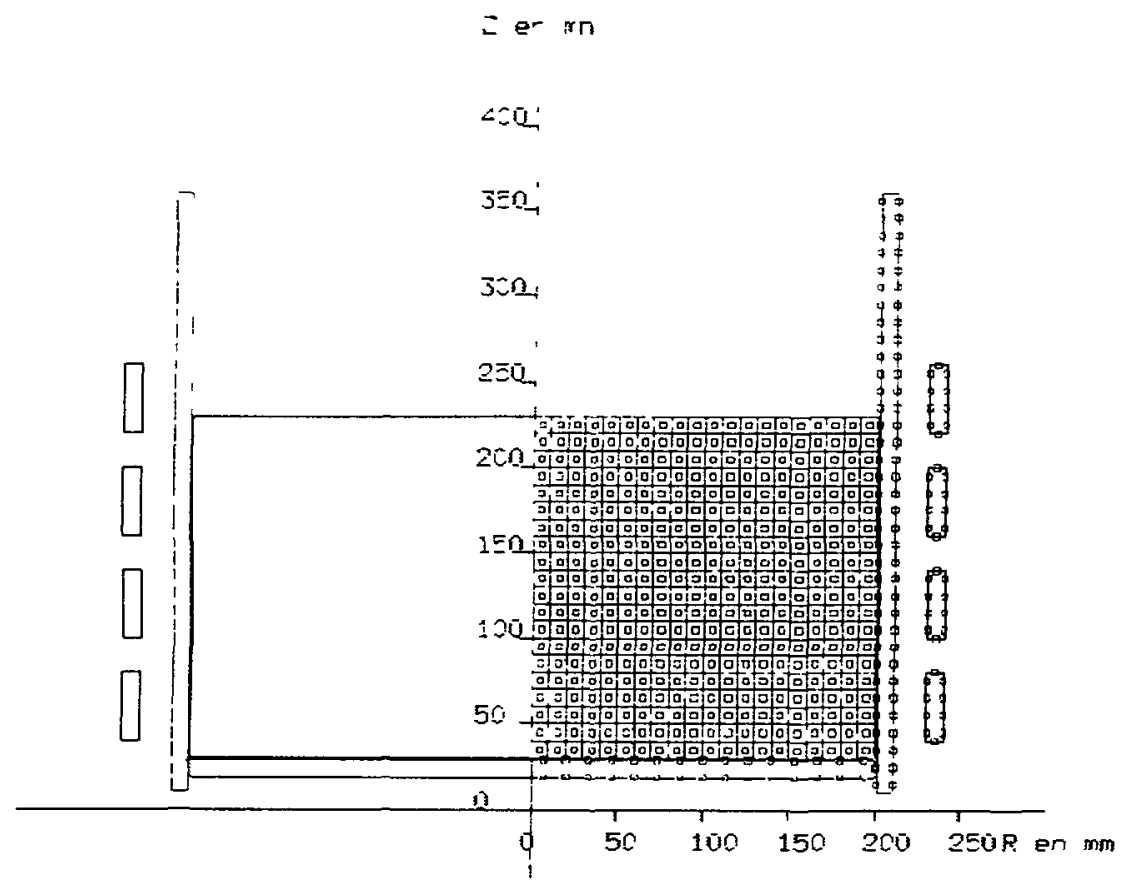

Fig. 6. - Représentation en coupe verticale du dispositif, configuration et maillage du modèle électromagnétique.

[Vertical section of the installation, mesh of electromagnetic model.] 


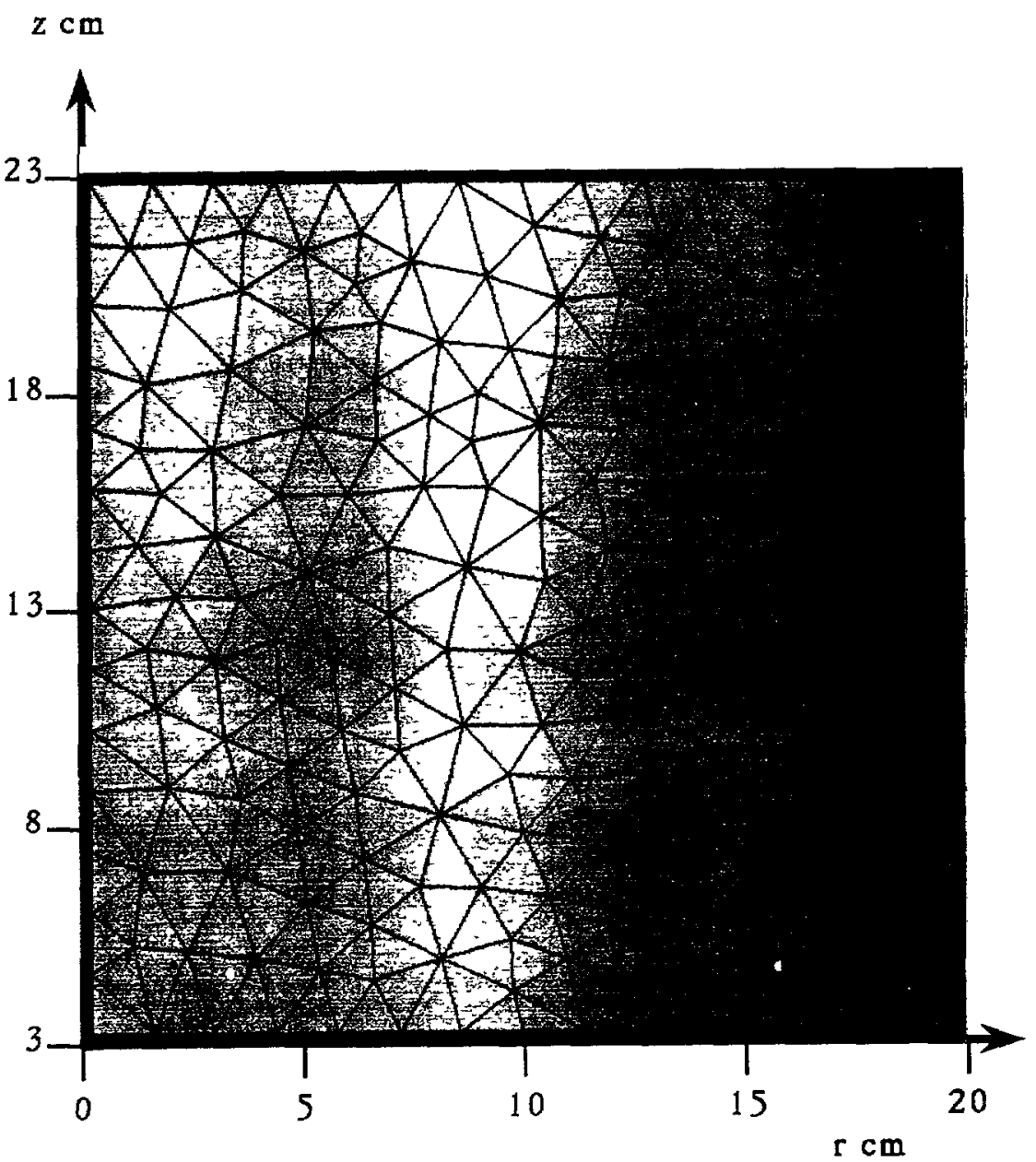

Fig. 7. - Maillage du modèle thermique de la région occupée par le verre.

[Thermal mesh of oxide domain.]

puissance ne tient compte que des paramètres de nature électromagnétique (proximité de la sole par exemple) (Fig. 10a). Or le refroidissement dû à la proximité d'éléments refroidis (creuset) ou au rayonnement influe sur la répartition des sources de puissance par l'intermédiaire de la conductivité électrique (Fig. 10b).

\section{Conclusion.}

En creuset froid, lorsque la conductivité électrique d'un matériau dépend fortement de la température, il est inconcevable de modéliser les phénomènes électromagnétique et thermique de manière découplée. Cependant, le couplage fort consistant à résoudre simultanément tous les phénomènes avec la même méthode numérique est pénalisant car il ne permet pas d'optimiser la place mémoire nécessaire et le temps de calcul. Cette dernière considération acquiert une importance particulière lorsque le nombre d'inconnues par nœuds augmente et 


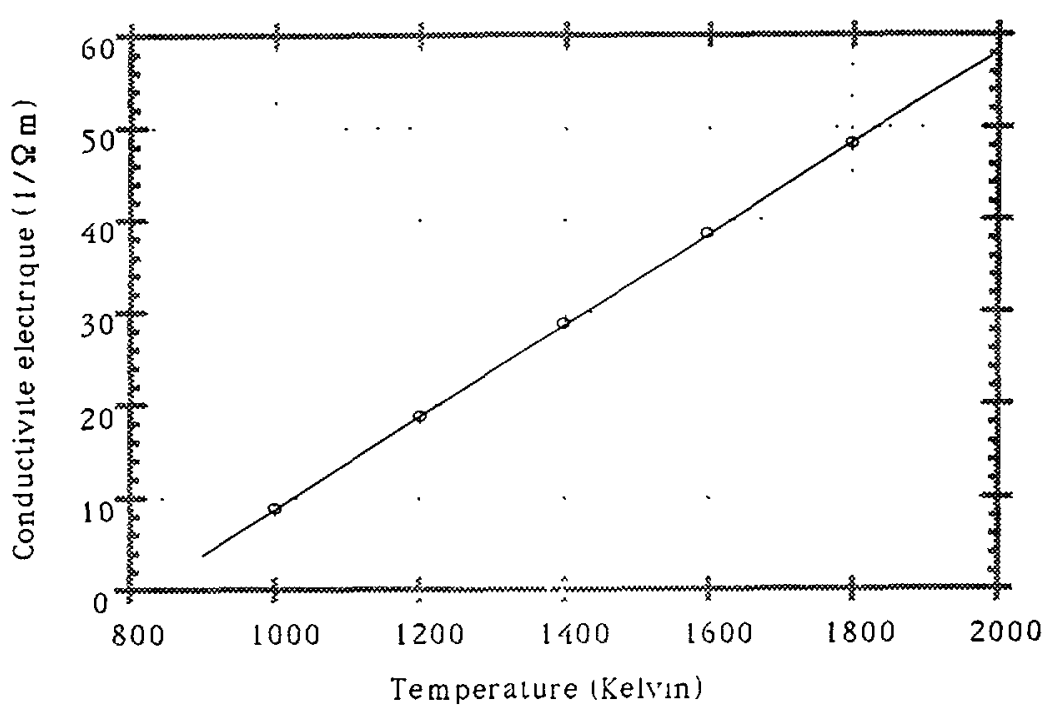

a) Conduchivile electrique

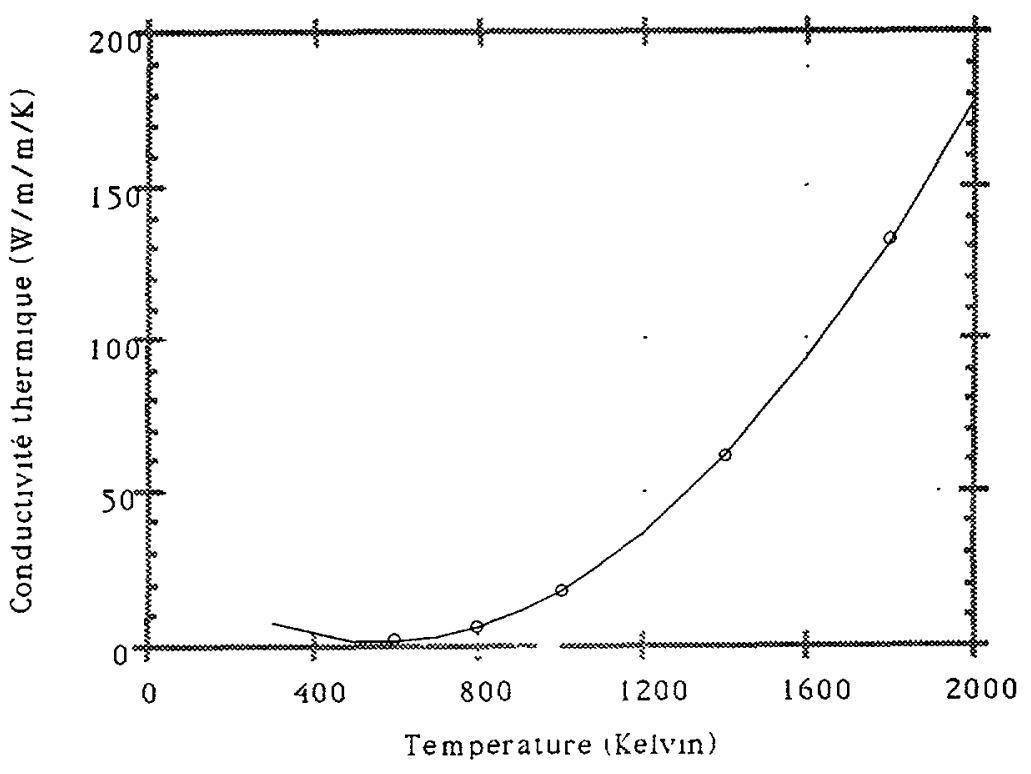

b) Conductsile ther mique

Fig. 8. - Modèles des propriétés physiques : a) conductivité électrique en fonction de la température, b) conductibilité thermique en fonction de la température.

[Physical properties: a) function of the electrical conductivity with respect to the temperature; b) function of thermal the conductivity with respect to the temperature.] 


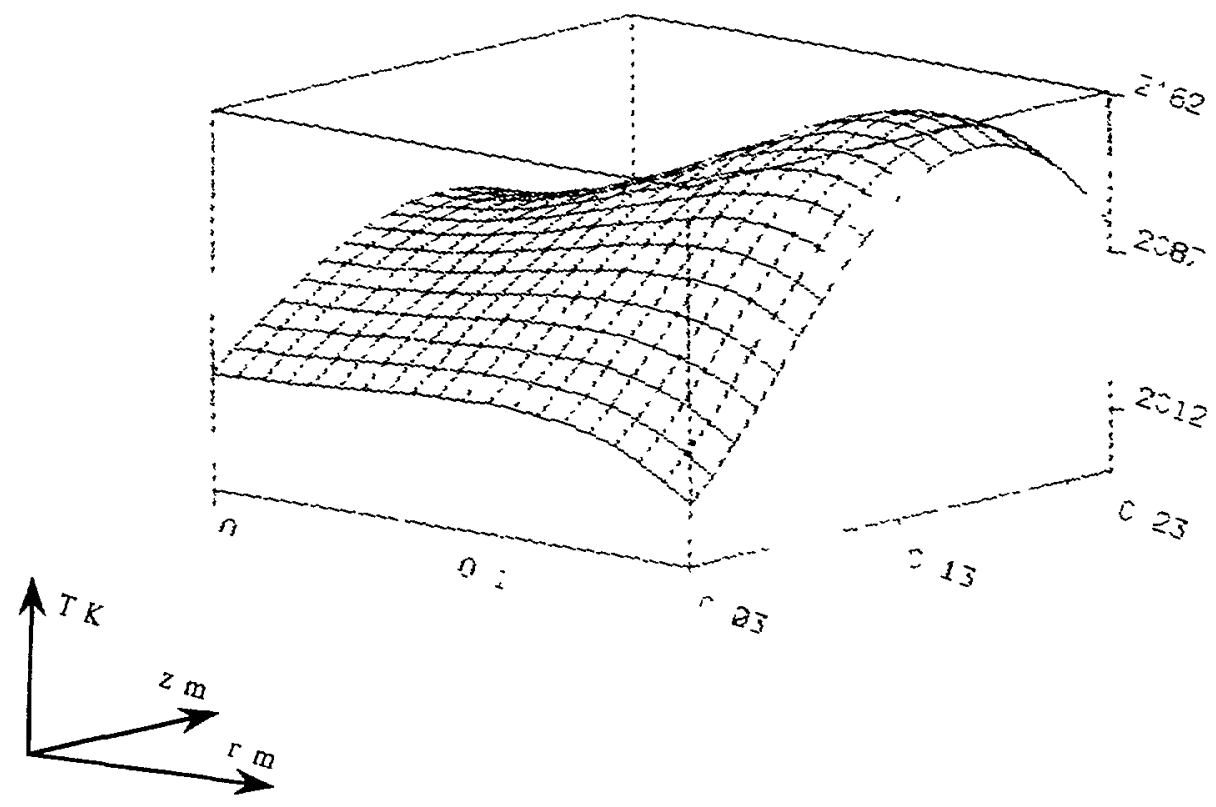

a)

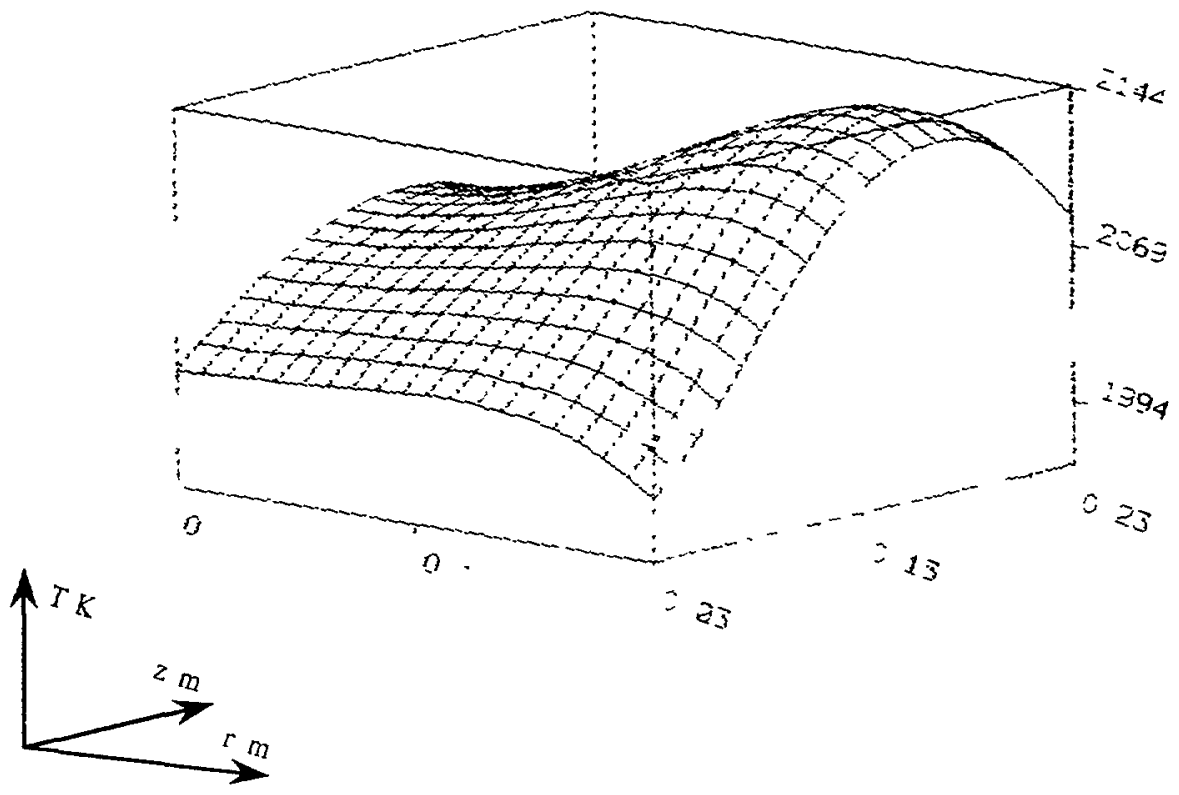

b)

Fig. 9. - Températures obtenues pour un courant inducteur de $750 \mathrm{~A}$ : a) sans couplage ; b) avec couplage.

[Temperature calculated with a $750 \mathrm{~A}$ coil current : a) without coupling ; b) with coupling.] 


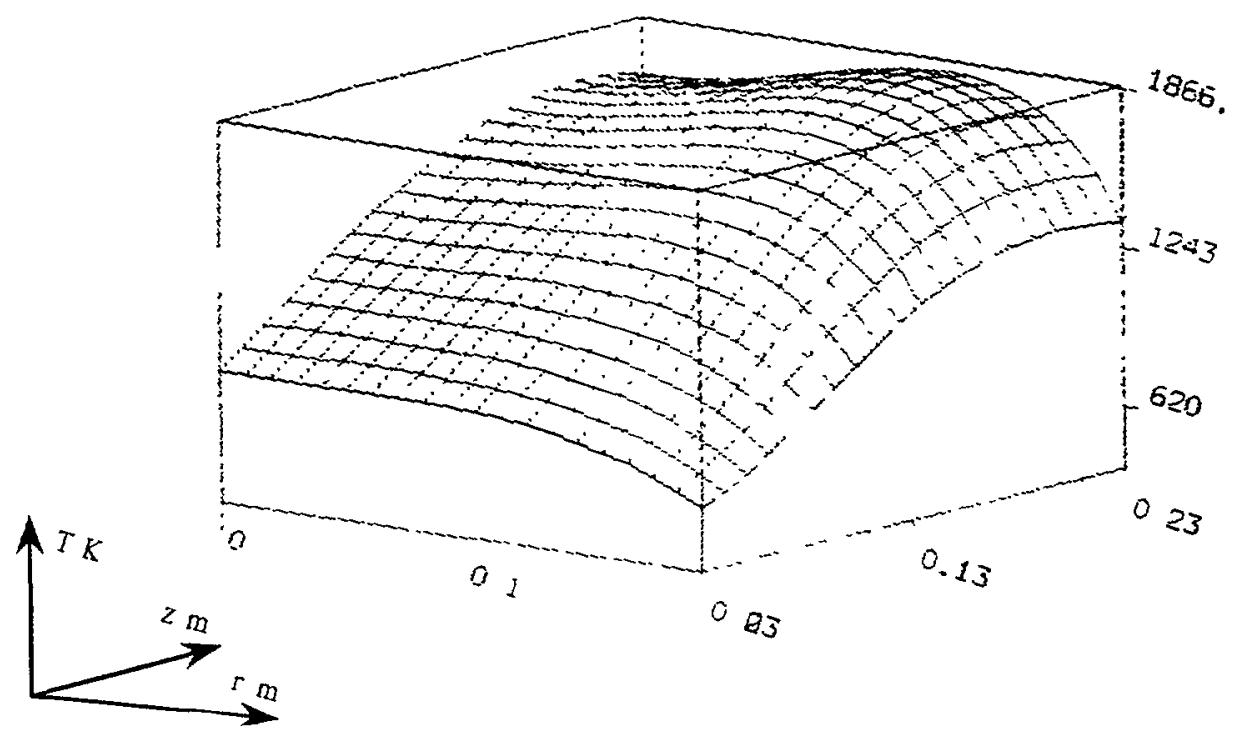

a)

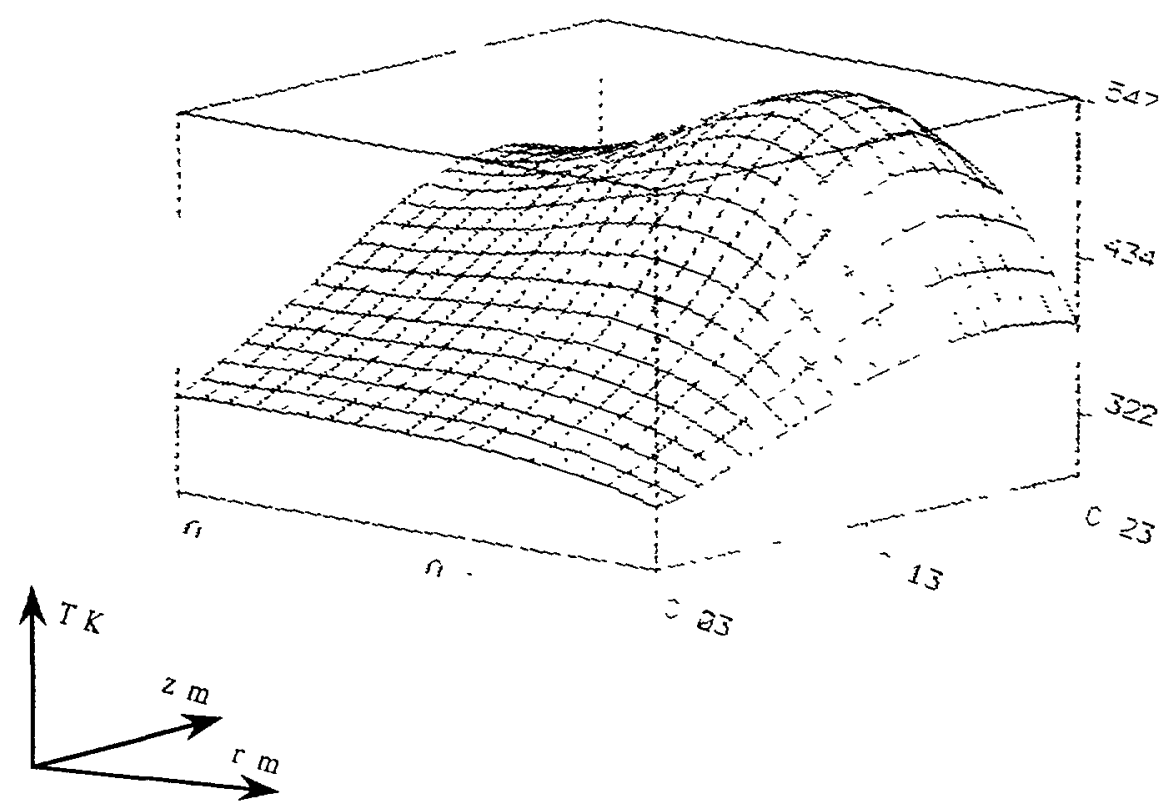

b)

Fig. 10. - Températures obtenues pour un courant sous dimensionné de $600 \mathrm{~A}$ : a) sans couplage, le bain de verre s'échauffe. La puissance Joule dans le verre est de $58,5 \mathrm{~kW}$; b) avec couplage, le bain de verre fige. La puissance Joule dans le verre est de $3,5 \mathrm{~kW}$.

[Temperature calculated with a $600 \mathrm{~A}$ coil current $:$ a) without coupling, the glass temperature raises. The Joule power in the glass is $58.5 \mathrm{~W}$; b) with coupling, the glass temperature decreases. The Joule power in the glass is $3.5 \mathrm{~kW}$.] 
que les géométries étudiées sont tridimensionnelles et complexes. Le logiciel Malice offre un bon compromis. Son algorithme itératif couple la méthode intégrale et la méthode des éléments finis pour modéliser les divers phénomènes intervenant dans ce procédé.

Sa conception évolutive permet de diversifier les phénomènes résolus en couplage avec l'électromagnétisme. 11 permettra à terme de dimensionner les installations creuset froid selon le matériau à élaborer, en tenant compte de ses propriétés physiques ou des exigences thermique et hydrodynamique nécessaires au cycle d'élaboration.

\section{Bibliographie}

[1] Massé Ph., Fautrelle Y., Gagnoud A., Coupled Methods for 3D Coupled Problems 10 Years of Experiments in MHD, IEEE Trans. Magn., à paraître.

[2] Gagnoud A., LeClerce I., Electromagnetic Modelling of Induction Melting Devices in Cold Crucibles, IEEE Trans. Magn. 24 (1988) 573-575.

[3] MASsÉ Ph., Modelling of continuous media methodology and CAD of Finite Element Programs, INTERMAG Conference, Hamburg, F.R.G. (1984).

[4] NarotTam P. Bansal, Doremus R. H., Handbook of glass properties (Academic Press, 1986).

[5] Scholze H., Le verre : nature, structure et propriétés, 2nde édition (1980) Institut du Verre.

[6] Patankar S. V., Numerical heat and fluid flow, Series in computationnal methods in mechanics and thermal seiences (1980). 